A mobile mediation tool for improving interaction between depressed individuals and caregivers

Sheng-Hsiang Yu, Li-Shan Wang, HaoHua Chu, Sue-Huei Chen, Cheryl ChaiHui Chen, Chuang-Wen You \& Polly

\title{
Huang
}

\section{Personal and Ubiquitous \\ Computing}

ISSN 1617-4909

Volume 15

Number 7

Pers Ubiquit Comput (2011) 15:695-706

DOI 10.1007/s00779-010-0347-z

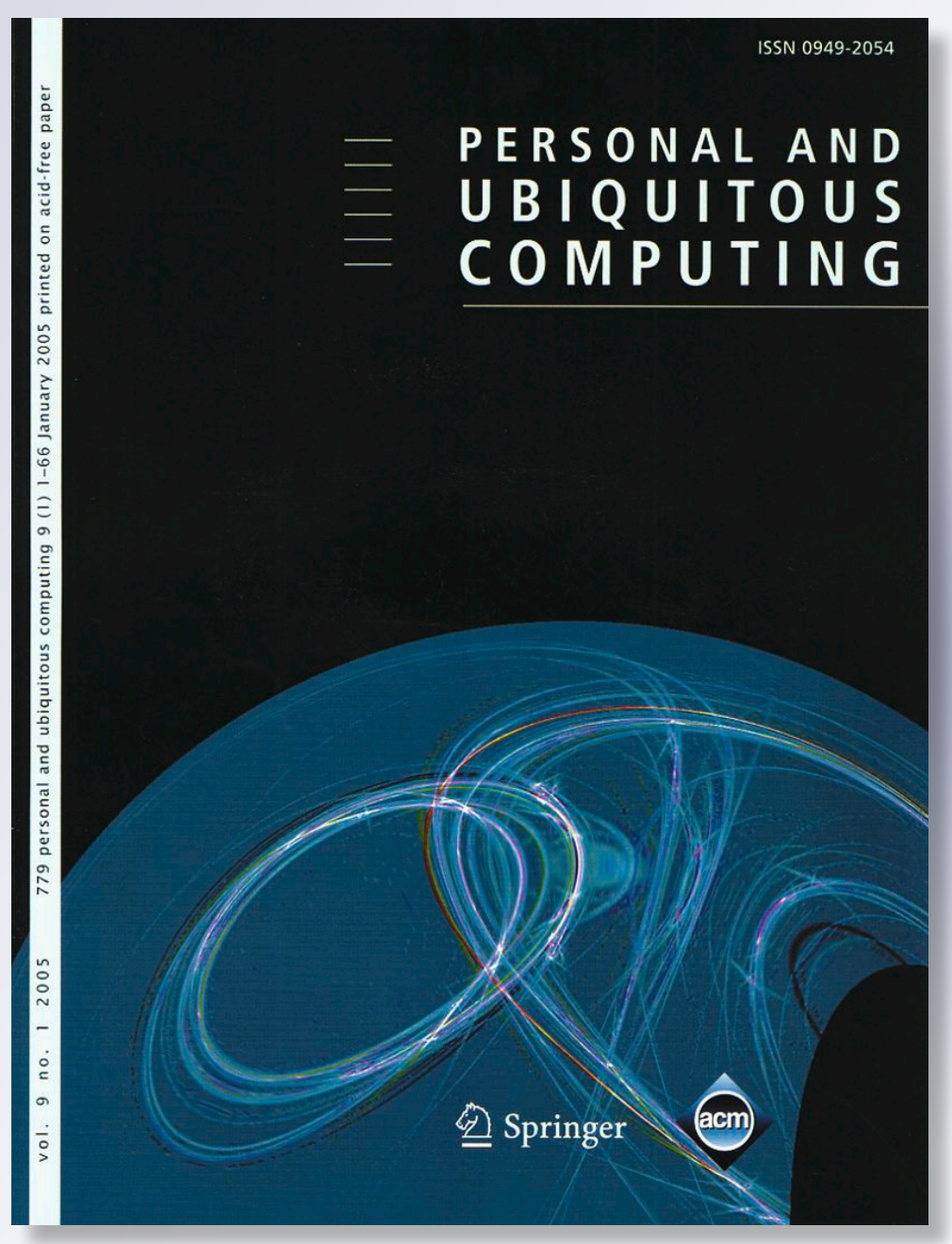

望 Springer 
Your article is protected by copyright and all rights are held exclusively by Springer-Verlag London Limited. This e-offprint is for personal use only and shall not be self-archived in electronic repositories. If you wish to selfarchive your work, please use the accepted author's version for posting to your own website or your institution's repository. You may further deposit the accepted author's version on a funder's repository at a funder's request, provided it is not made publicly available until 12 months after publication. 


\title{
A mobile mediation tool for improving interaction between depressed individuals and caregivers
}

\author{
Sheng-Hsiang Yu $\cdot$ Li-Shan Wang $\cdot$ \\ Hao-Hua Chu $\cdot$ Sue-Huei Chen · Cheryl Chai-Hui Chen • \\ Chuang-Wen You $\cdot$ Polly Huang
}

Received: 26 March 2010/Accepted: 12 November 2010/Published online: 10 December 2010

(C) Springer-Verlag London Limited 2010

\begin{abstract}
Depression, a common mental disorder, significantly affects an individual's ability to live a normal life. Excessive reassurance-seeking is a common interpersonal behavior characteristic of depression and often leads to negative interaction between caregivers and depressed individuals, who seek excessive assurance from the
\end{abstract}

\section{S.-H. Yu · S.-H. Chen}

Department of Psychology, National Taiwan University,

Taipei, Taiwan

e-mail: yu.shenghsiang@gmail.com

S.-H. Chen

e-mail: shchen@ntu.edu.tw

\section{L.-S. Wang · H.-H. Chu $(\bowtie)$}

Department of Computer Science and Information Engineering,

National Taiwan University, Taipei, Taiwan

e-mail: hchu@csie.ntu.edu.tw

L.-S. Wang

e-mail: r95922069@ntu.edu.tw

S.-H. Chen

Department of Psychiatry,

National Taiwan University Hospital, Taipei, Taiwan

C. C.-H. Chen

School and Graduate Institute of Nursing,

National Taiwan University, Taipei, Taiwan

e-mail: cherylchen@ntu.edu.tw

\section{C.-W. You}

Research Center for Information Technology Innovation,

Academia Sinica, Taipei, Taiwan

e-mail: cwyou@citi.sinica.edu.tw

\section{P. Huang}

Department of Electrical Engineering,

National Taiwan University, Taipei, Taiwan

e-mail: phuang@cc.ee.ntu.edu.tw caregivers at inappropriate times, such as when caregivers are busy. Such negative interaction results in elevated feelings of burden for both caregivers and care receivers. However, maintaining a good relationship between depressed individuals and caregivers is crucial to overcoming difficult times during depression. Therefore, we propose a mobile care mediation system that enables depressed individuals and caregivers to share mood and availability information, thus improving communication in caregiving and care-receiving and reducing the caregiving burden.

Keywords Mobile computing · Awareness .

Depression · User interface

\section{Introduction}

Depression has become a common mental disorder, affecting an increasing number of individuals in recent decades. As a mental disorder, major depressive disorder is a leading cause of disability in the United States [1]. According to the Global Burden of Disease Estimate (GBD) of WHO [2], major depressive disorder (MDD) places an enormous burden on society and ranks as the fourth leading cause of burden among all diseases. If the current trend for demographic and epidemiological transitions continues, then the burden of MDD will become the second leading cause of DALYs (Disability-Adjusted LifeYear) lost after ischemic heart disease for both sexes worldwide by the year 2020 [3]. The Department of Health, Executive Yuan (Taiwan) estimates that approximately one million individuals currently suffer from depression in Taiwan. Moreover, suicide is one of the top ten causes of death in Taiwan [4], and also most suicide victims suffer from major depression around the time of death $[5,6]$. 
Information technology can be used to help support current depression treatment. Computer-based software tools have been designed to help depressed individuals follow a specific cognitive behavioral therapy [7, 8]. Online treatments for depression (web-based applications or web sites) have been developed to enable self-help treatment for depression and anxiety [9]. Palmtop- or PDAbased cognitive behavioral therapy treatments have also recently appeared that provide patients with easier access to treatment (e.g., at work or at school) [10, 11].

This work targets excessive reassurance-seeking behavior per the interpersonal model of depression. Reassurance seeking behavior significantly exacerbates and prolongs depressive moods and symptoms. Excessive reassurance-seeking is a common characteristic of interpersonal behavior among depressed individuals [12]. Depressed individuals tend to seek reassurance from others to alleviate their doubts about their own worth and to obtain support and care from their caregivers (e.g., significant others). Therefore, depressed individuals always face a dilemma, in that they both need and doubt the assurance of others. However, this reassurance-seeking behavior is so repetitive that the caregivers may finally become frustrated and irritated. Perceiving an increased burden in caring for depressed individuals, the caregivers may reject their requests for help. Consequently, depressed individuals become ever more depressed, since they cannot obtain assurance from caregivers to help them get through difficult periods. Therefore, excessive reassurance-seeking leads to a vicious cycle of negative interaction between depressed individuals and caregivers $[13,14]$.

\subsection{Opportunities for mobile computing}

Mobile computing technology can help in mediating care interaction (i.e., caregiving and care-receiving) between depressed individuals and caregivers. Mobile technology enables timely exchanges of status information between depressed individuals and caregivers and provides mutual awareness of each other's availability and mood. Such awareness and understanding is often an important prelude to a satisfactory caregiving and care-receiving communication. For example, a depressed individual can know when a caregiver is or will be available for caregiving. Meanwhile, a caregiver can observe when a depressed individual enters a negative mood and can thus give care to the depressed individual at the needed moments. Second, mobile technology serves as a virtual agent, suggesting activities to distract depressed individuals from excessive reassurance-seeking behavior. At the same time, this virtual agent can also offer advice to a caregiver on how to provide care to the depressed individuals.

\subsection{Application scenario}

We motivate this work with the following excessive reassurance-seeking scenario involved a depressed individual and her caregiver:

Miss A is a campus student and has depressive symptoms. She also has a boyfriend who is also a campus student. They take different courses during the day and sometimes take part in different afterclass activities. In the evening, they go back to their own homes. Since they are often not together, they communicate via mobile phones and desktop computers. During the day time when they do not have access to their own computers, they usually call each other. She always calls her boyfriend whenever she feels depressed, and asks him about her worth, whether he truly loves her. However, she does not know whether her boyfriend will be free to talk to her. When her boyfriend is busy and unable to accept the call, she calls him repeatedly, often losing her temper. She blames him for not accepting the call as soon as possible. She doubts that her boyfriend cares enough for her. Her boyfriend also feels guilty and frustrated, because his comfort to her does not fulfill her needs. Actually, her reassurance-seeking behavior and the resulting negative interactions over the call have made him afraid to talk to her.

\subsection{Mobile care mediation system}

In this work, we propose a mobile phone-based system to mediate caregiving and care-receiving between caregivers and depressed individuals by sharing their availability and mood information, thus reducing the probability of untimely calls for reassurance-seeking in the unmediated scenario described above. Additionally, the proposed phonebased care mediation system gives behavioral suggestions, in the form of distraction exercises, when a depressed individual enters a negative mood.

A mobile phone-based approach has several advantages over the traditional paper-based approach. (1) A mobile phone periodically reminds users to record their mood and availability anytime, anywhere; whereas the traditional paper-based approach requires users to remember when to record their mood and availability on booklets and often leads to missing entries. (2) Since a mobile phone is the primary calling device for most people, sharing availability and mood over a mobile phone enables a depressed individual and a caregiver to conveniently check the appropriate timing of a call, i.e., prior to placing the actual call on the phone. 
Current instant messaging (IM) and short messaging services (SMS) enable users to update and publish socalled presence information (i.e., status) that indicates their willingness or availability for communication [15]. Studies have shown that presence information is a key component in instant messaging applications [16]. This study further extends this presence information to include rich context information that facilitate better caregiving and carereceiving communication between depressed individuals and their caregivers.

The proposed study is executed in two stages. In the first stage, we create and evaluate phone-based ESM (Experience Sampling Method), in which the phone is preprogrammed to periodically prompt its user to answer questions about his or her current mood and experiences. Based on the answers to these questions, the phone-based ESM system recommends activities that will be helpful in distracting the user from his or her negative mood. In the second stage, we prototype and evaluate a phone-based care mediation system, in which care-receiver/caregiver pairs use phones to share their mood and availability information. The phone-based ESM system, created in the first stage of this study, is used in the second stage to sample and measure user experiences with the phone-based care mediation system.

\subsection{Organization}

The remainder of this paper is organized as follows. In Sect. 2 we discuss related work, and in Sect. 3 we present the phone-based ESM system, or the first phase of this study, including its design, implementation, and evaluation. In Sect. 4 we describe the mobile phone-based care mediation system, or the second phase of this study, covering its design and implementation. In Sect. 5 we describe the user study evaluation of this mobile care mediation system, and in Sect. 6 we discuss the lessons learned. We draw conclusions in Sect. 7 and discuss future research directions of the mobile care mediation tool.

\section{Related work}

In the mobile computing research literature, there is great interest in obtaining in situ data and in remote health care (both physical and mental). Much mobile technology work in experience sampling focuses on sensing and uses sensors to trigger experience sampling studies on mobile devices. The context-aware experience sampling (CAES) tool [17] utilizes environmental sensors and biosensors to trigger sample collections from specific circumstances (e.g., the person is near a store). MyExperience [18] incorporates sensors into experience sampling tools to help evaluate user interaction with mobile devices and applications, and supports automatic logging of device states and sensor data. SocioXensor [19] applies logging sensors (such as conversation, location, and Bluetooth proximity) and usersubjective data to evaluate social phenomena.

Some studies focus not only on collecting in situ data, but also support interaction with professionals and researchers. Momento [20] is a client-server architecture that uses SMS/MMS as its communication medium. A mobile client gathers data and uploads them to a server, enabling the server-side researcher to send the information required by the mobile user. MAHI [21] is an experience sampling tool with a blood glucose meter and also captures voice notes and photographs. Users also share their records via a website, and have access to diabetes educators. The proposed mobile care mediation tool focuses on enhancing both the understanding and the quality of interaction between depressed individuals and caregivers, but not between users and professionals.

Caring for a loved one or for oneself is essential for a good quality of life. Many studies have designed systems that display a user's status to family members or the user. In order to provide a sense of family connectedness, the digital family portrait system [22] displays information in a digital photo frame about the day-to-day activities of a distant family member who is not living with the family. The CareNet Display [23] adopts an augmented digital picture frame to provide care to an elder within a care network. This work helps care network members to coordinate care activities, ensuring that the elder person receives the required care. The above two studies focus on providing awareness information about others. The UbiFit Garden [24] encourages a user to increase his/her own activity by providing feedback in a virtual garden of a mobile home screen. The Playful toothbrush [25] embeds a behavioral intervention when brushing teeth among children in kindergarten. A virtual-teeth game is presented to suggest brushing technique and to encourage children to brush their teeth properly. While these two projects help users to be more aware of their own behaviors, the mobile care mediation tool makes the user aware of the other user's status and suggests actions for the user to perform. Therefore, the proposed tool incorporates an awareness display interface that is designed to show the status of both users on a mobile phone home screen.

Ubiquitous psychotherapy [26] is a general tool being developed for mobile devices; the tool tries to assist therapists and patients using cognitive behavior therapy (CBT). It provides a personalized homework tool for patients so that therapists can assign and adjust artifacts for each patient during CBT treatment. Blues Begone [8] is a computer-based software tool to help depressed individuals to recover from depression. The software provides a 


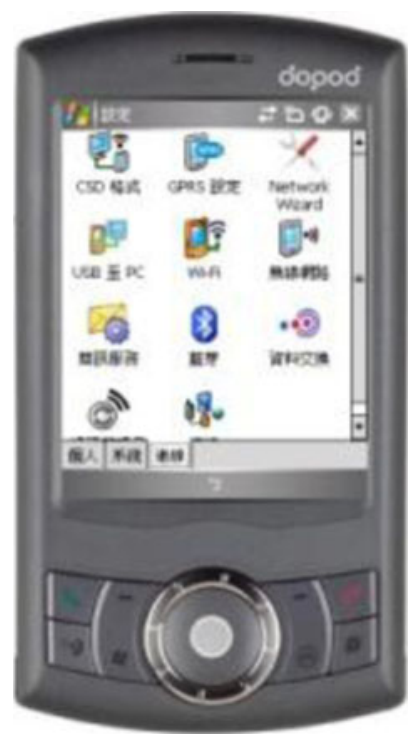

Fig. 1 Mobile components of ESM tool

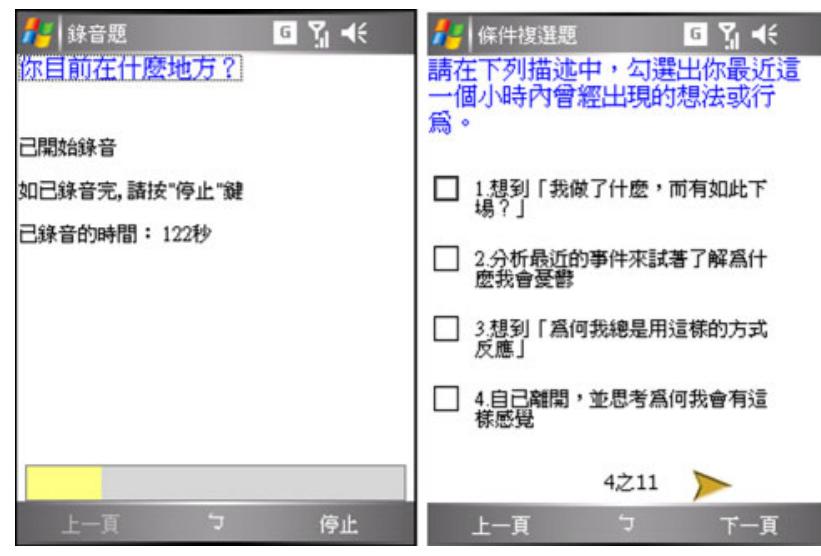

Fig. 2 Prompted questionnaire screenshots

structured daily program of cognitive behavioral knowledge and techniques to discover and rectify faulty thoughts, beliefs, and assumptions. The above two studies focus on CBT treatment and lack support from social environments, but the mobile care mediation tool is also designed to help caregivers give support to the depressed individuals.

\section{Phone-based ESM system}

The phone-based ESM system was prototyped on a Dopod p800w smart phone, as illustrated in Fig. 1. A screenshot of an ESM question in Chinese is shown in Fig. 2. To simplify user entries on a phone and reduce the amount of time needed to answer these questions, input for the ESM questions is multi-modal: radio buttons for single-choice questions, combo box for multiple-choice questions, and voice input for open questions. Researchers can dynamically adjust the frequency of the experience samples. To notify users to answer the questions, the ESM system uses the existing phone profile setting. The three possible notification methods are (1) sound, (2) vibration, and (3) no alarm. The ESM system automatically logs timestamps whenever users complete the questionnaires.

The ESM system calculates a mood score from the answers to the questions. When the mood score indicates a depressed mood, the ESM system suggests activities that will be helpful in distracting the depressed patients from thinking too much: (1) taking a walk, (2) running or bicycling, (3) shopping, (4) listening to music, and (5) performing a relaxation exercise. Users are asked to perform one of these activities for at least $15 \mathrm{~min}$. The system then checks user compliance by asking the depressed individuals whether the suggested activities were carried out.

\subsection{World visit game}

One of the main challenges for any ESM system to work as an effective data collection tool is to get the user to promptly fill out the questionnaires. To motivate users to fill out the questionnaires, our ESM tool introduces a game that rewards users after they complete questionnaires or perform the recommended activities. Figure 3 shows screenshots of the world visit game. At the start of the game, a user selects a character (boy or girl) and three places he or she wants to visit. Every place involves walking 50 steps, after which the user visits the next place.

The game rewards are given based on the following rules: (1) A user receives one step and one milk bottle after completing a questionnaire. (2) A user receives two steps and two milk bottles after performing a distraction activity. (3) A user rolls dice after gathering three milk bottles. The user's character walks extra steps according to the dice results. The third reward rule introduces a probabilistic factor into the game, which makes the game more fun.

\subsection{ESM user study}

The user study was conducted in collaboration with the Department of Psychology of the National Taiwan University (NTU). The participants were NTU students and were recruited using an Internet advertisement. Students with depressive symptoms were screened using the Beck Depression Inventory-II (BDI-II) [27]. ${ }^{1}$

\footnotetext{
${ }^{1}$ The BDI-II is a 21-item, self-reported measure of the severity of depression symptoms. Items of various severities of depression symptoms are rated on a 4-point Likert scale, with total scores ranging from 0 to 63 .
} 
Fig. 3 World visiting game interface
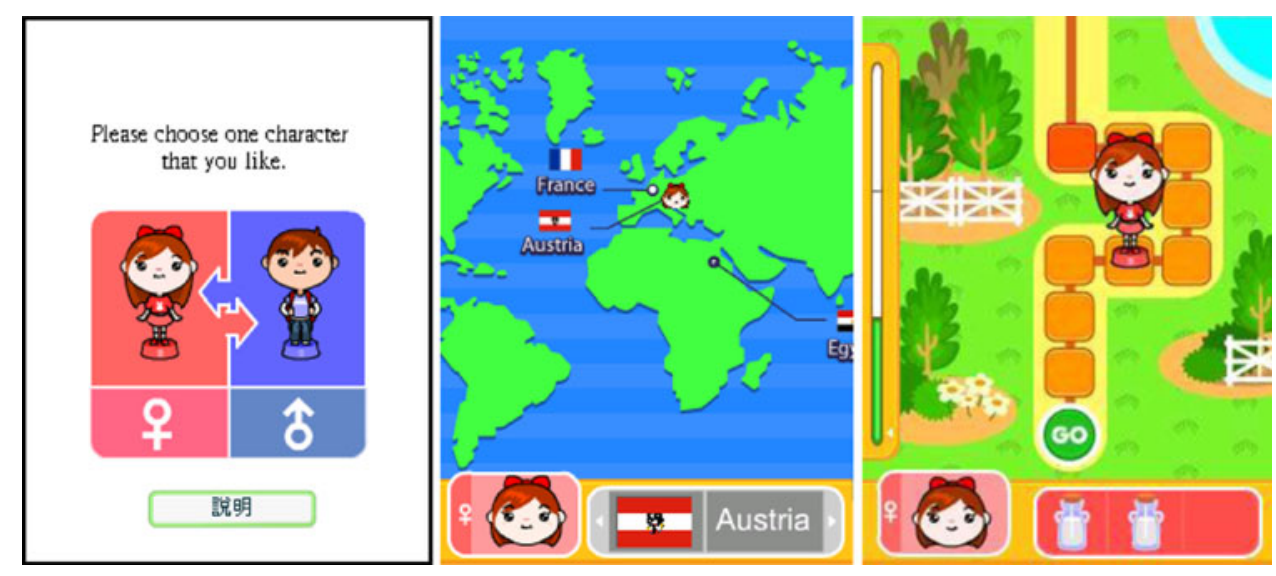

For the purpose of comparison with phone-based ESM, we developed a traditional paper-based ESM. An automatic calling tool, implemented on top of Skype, was used to call and notify users to fill out booklet questionnaires. The users carried the booklet questionnaires throughout the day.

\subsubsection{Procedure}

All of the students were paid NT $\$ 150$ (about US\$5) for participating in the pre-ESM assessment (BDI-II, Negative Mood). The participants with a BDI-II score greater than 15 were invited to participate in an 8-day ESM experiment and post-ESM assessment and were paid a further NT $\$ 500$ (about US\$16). Each participant was randomly assigned to one of three experimental groups: (1) Group 1, which used the phone-based ESM without the world visit game; (2) Group 2, which used the phone-based ESM with the world visit game; and (3) Group 3, which used the traditional paper-based ESM. Due to time and resource limitations, the numbers of participants in each group were not equal. Group 1 had 8 participants, Group 2 had 9 participants, and Group 3 had 11 participants. Table 1 lists the statistics (mean and standard deviation) of the participants. The participants with high BDI-II scores also had high negative moods.

Table 1 Participant characteristics

\begin{tabular}{lllc}
\hline & \multicolumn{3}{l}{ Depressed participants $(n=28)$} \\
\cline { 2 - 4 } & Mean & SD & $n$ \\
\hline Number of females & & & 21 \\
BDI-II $^{\mathrm{a}}$ & 21.29 & 5.73 & \\
Negative mood for previous week $^{\mathrm{b}}$ & 17.96 & 3.24 &
\end{tabular}

a BDI-II scores range from 0 to 63

b Negative mood is assessed by six items on a 5-point Likert scale. The score ranges from 5 to 30

\subsubsection{Measurement}

The questionnaires were designed to measure mood fluctuation, ruminative responses, contextual information about the user's current location, the activities that the user has carried out, and interpersonal behavior (e.g., seeking assurance or support from others). A questionnaire was triggered five times per day-an average frequency of every two and half hours-to sample user experience. Twice a day, activities were recommended to participants following questionnaires: once before and once after $5 \mathrm{pm}$.

The following items were measured in this study.

- Compliance rates for the three groups of participants in completing the ESM questionnaires or recommended activities. The compliance rate was calculated as the number of completed questionnaires and activities performed over the total number of questionnaires and activities requested.

- User experience with the ESM system.

\subsubsection{Results: average compliance rate}

A total of 667 ESM samples were collected from 28 participants during the 8-day ESM study. Table 2 shows the mean and standard deviation of the compliance rates for the completion of questionnaires and performance of the recommended activities. There are two main findings. (1) For questionnaire compliance, Group 2 (phone-based ESM

Table 2 Compliance rates of three groups

\begin{tabular}{|c|c|c|c|c|c|c|}
\hline & \multicolumn{2}{|c|}{$\begin{array}{l}\text { Group 1 } \\
(n=8)\end{array}$} & \multicolumn{2}{|c|}{$\begin{array}{l}\text { Group } 2 \\
(n=9)\end{array}$} & \multicolumn{2}{|c|}{$\begin{array}{l}\text { Group } 3 \\
(n=11)\end{array}$} \\
\hline & Mean & SD & Mean & SD & Mean & SD \\
\hline Questionnaires & 0.55 & 0.13 & 0.67 & 0.12 & 0.59 & 0.15 \\
\hline Activity & 0.47 & 0.17 & 0.48 & 0.17 & 0.40 & 0.22 \\
\hline
\end{tabular}


Table 3 User experience feedback on the ESM study

\begin{tabular}{|c|c|c|c|c|}
\hline & \multirow{2}{*}{$\begin{array}{l}\text { Paper-based }(n=11) \\
\text { Mean (SD) }\end{array}$} & \multirow{2}{*}{$\begin{array}{l}\text { Phone-based }(n=17) \\
\text { Mean (SD) }\end{array}$} & \multicolumn{2}{|c|}{ Group difference } \\
\hline & & & $t$ & $p$ \\
\hline Complexity $^{\mathrm{a}}$ & $0.92(0.30)$ & $0.47(0.51)$ & -2.54 & $.02 *$ \\
\hline Interference $^{\mathrm{b}}$ & $1.72(0.64)$ & $1.11(0.48)$ & -2.85 & $.01 * *$ \\
\hline Positive attitude ${ }^{c}$ & $1.67(0.50)$ & $2.18(0.73)$ & 2.14 & $.04 *$ \\
\hline
\end{tabular}

a The "perceived complexity of the questionnaire" was assessed with one question: $0=$ not at all, $1=$ a little, and $2=$ very complex

b "Interference in daily life" was assessed with two questions. The average scores reported here ranged from 0 to $3.0=$ not at all, $3=$ significant interference

c The "attitude toward ESM" was assessed with two questions. The average scores reported here ranged from 0 to 3 . $0=$ not positive or interesting, 3 = very positive or interesting

with the game reward) performed the best at $67 \%$, followed by Group 3 (paper-based ESM) at 59\%, and lastly Group 1 (phone-based ESM without the game reward) at 55\%. On the activity compliance, Group 2 again performed the best at $48 \%$, followed by Group 1 at $47 \%$, and finally Group 3 at $40 \%$. Pairwise comparison reveals differences in the questionnaire compliance rate between Groups 2 and 1 $(t=1.98, p<.07)$, which suggests that game reward improved the user compliance for phone-based ESM. (2) There is little difference in user compliance between Groups 1 (phone-based ESM) and 3 (paper-based ESM); this suggests that without the game reward to motivate participants to perform phone-based ESM, the result is no better than that of the traditional paper-based ESM.

\subsubsection{Results: user experiences}

Table 3 shows the comparison of user experience results between the phone-based and paper-based ESM systems. No subjects dropped out in the phone-based ESM, while two participants in the paper-based ESM did not return the ESM questionnaires and gave no reason why. Paper-based participants responded that questionnaire design was more complex than the phone-based participants. Interference in daily life was more significant in paper-based group. Phone-based participants had a more positive attitude toward the ESM study than the paper-based participants. Even though none of the users had any experience using touch screen phones, they replied that replying to the ESM questionnaires was very straightforward.

\section{Mobile care mediation system}

This mobile care mediation system was prototyped on the HTC Touch smart phone shown in Fig. 5 using text messaging (SMS) and media messaging services (MMS). It included the phone-based ESM system, described in Sect. 3 , for data collection from users. Additionally, it

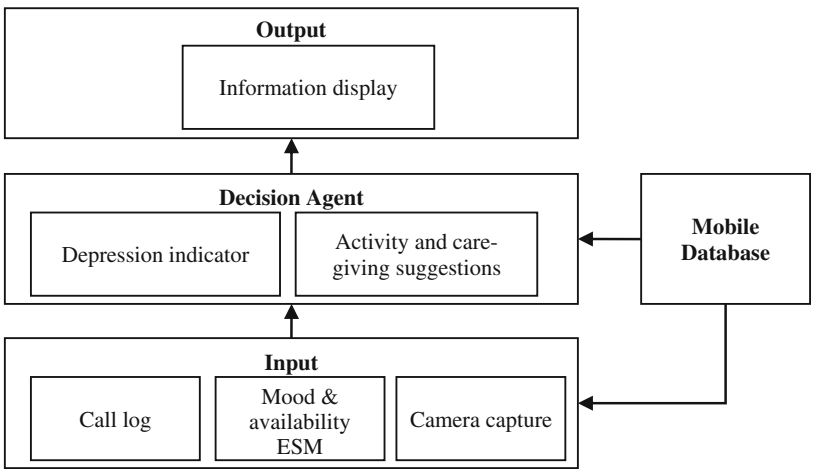

Fig. 4 Architecture of the mobile care mediation tool: input, decision agent, and output

incorporated two-way sharing of availability and mood information between the depressed individuals and their caregivers.

Figure 4 shows the three-tiered architecture of this mobile care mediation system, including the input layer (e.g., record mood), the decision agent, and the output layer (e.g., the display). These three layers are interconnected through a mobile database. The design and implementation details of the mobile database and each layer in the system architecture are described as follows.

\subsection{Mobile database}

An SQL Server 2005 Mobile Edition (SQL Mobile) database was used for local storage on PDA phones. The database stores input data such as mood, availability, and call time logs for each party. The decision agent queries the database to determine which information to show on the display screen, or to remind the user of a behavior suggestion if the logic condition set in the decision tree is true.

\subsection{Input}

Input refers to user interaction on the device, or user input when answering ESM questionnaires (such as 


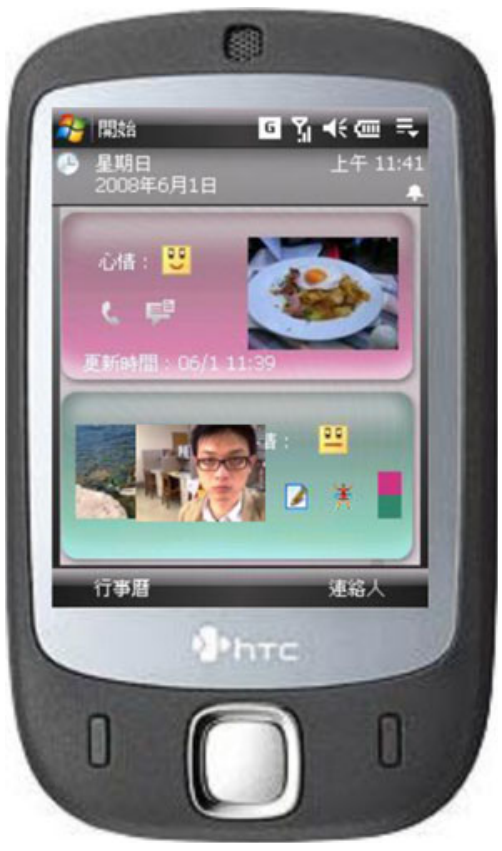

Fig. 5 Information display on an HTC Touch

mood). The display in Fig. 5 acts not only as an output display, but also as an input interface for users (depressed individuals and caregivers) to modify their availability status and to capture images. The mobile care mediation tool is designed around the user input to communicate between depressed individuals and caregivers. Both the depressed individuals and the caregivers need to input their mood and availability. Additionally, since most mobile phones have built-in cameras, users can easily capture self-portraits or the images of their environments that convey additional information about their current emotion, location, and activities. The system also logs any incoming or outgoing calls to or from a caregiver for analysis of the calling behavior of a depressed individual.

\subsection{Decision agent}

The decision agent handles input data, and acts as an agent in making decisions. The input mood-as derived from the questionnaires-is split into three types: (1) positive, (2) negative, and (3) depressed. The agent sets threshold levels for determining the level of the mood state. When the input data satisfies a given condition, the agent finds the output of that condition in the database, and then updates the display to give a suggestion to the depressed individual, such as encouraging him or her to perform distraction exercises rather than to stay in a negative mood, or notifies the caregiver with a suggestion on how to care for the depressed individual.

\subsection{Output}

The agent outputs its decision in three formats. In the first format, the output data is presented on the phone display shown in Fig. 5. The display contains mood emoticons, an availability status background, avatar images, and a few shortcut links. Second, the behavioral suggestions guide depressed individuals and caregivers to interact in the correct way. One behavioral suggestion is as follows: "You're feeling down. When we feel this way, we tend to look to caregivers for support, but they can't always be there for you. We suggest that you do a distraction activity which will help improve your mood". Third, output messages are sent to the other party, i.e., the caregiver, through SMS or MMS communication on his or her mobile phone while updating the care-receiver's mood, availability status, and avatar images.

\subsection{User interface}

The mobile care mediation tool was implemented using $\mathrm{C \#}, \mathrm{C}++$, and the Windows CE API. The tool can be deployed on Windows Mobile 5.0 devices (e.g., the Dopod P800W) as well as Windows Mobile 6.0 devices (e.g., the HTC Touch). The UI components of the mobile care mediation tool are described in detail below.

The interface was implemented as a plug into the phone's home screen, as illustrated in Fig. 5. The display screen layout is separated into two parts: the upper part for the other party, and the lower part for the user. The upper part contains mood, availability status, and photos provided by the other party. It also has two shortcut links, respectively, for calling and for sending messages to the other party. It also displays the timestamp of the latest information update by the other party. The lower part displays the user's own information, likewise including mood, availability status, and photos. It has four shortcut links: (1) a link to the SMS inbox, (2) a link to the self-report mood questionnaires, (3) a link to distraction activities, and (4) a link to change the user's availability status.

The information display is updated as new window messages are received, after which the display windows are repainted with updated information from the local database. The emoticons on display represent any of five levels: very happy, happy, normal, sad, and very sad, as illustrated in Fig. 6. The availability status is identified by the background color, where green signifies free and red signifies busy.

Users reply to ten questions to update the current mood status. These ten items are designed according to PANAS (Positive and Negative Affect Scale) [28] to identify the level of positive moods, negative moods, and depression. The mood level is determined after the questionnaire has 

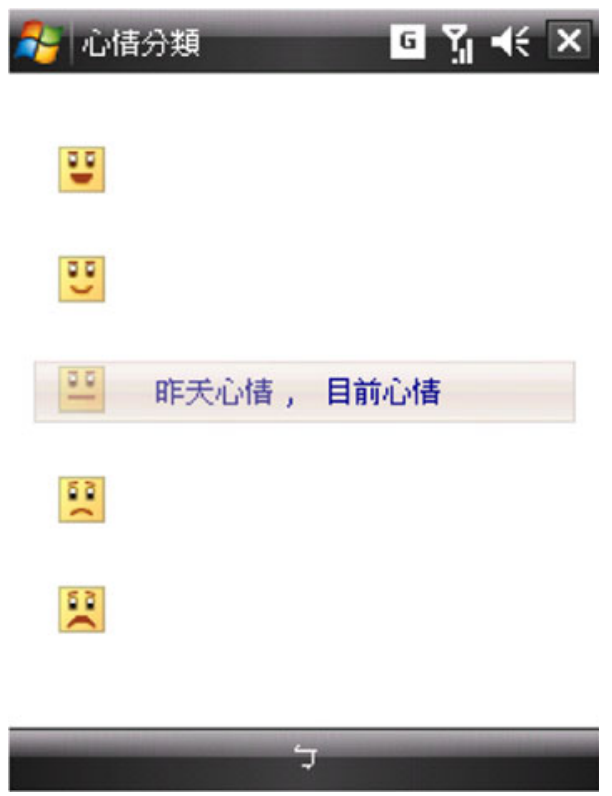

Fig. 6 Five mood levels

been completed, and the emoticon is updated to the display screen and sent to the other party to update the mood status.

To receive these SMS updated messages, the proposed system implements a message rule client (MAPI rules), which is a COM object that processes the incoming MAPI messages. The MAPI rules client intercepts all incoming SMS messages, and compares the messages with various rules. Messages tagged with "OInfo:" are processed by our custom program, while other messages are passed to the device's default Inbox. Intercepted messages about mood and availability are stored in the local database, and are also updated to the display with timestamps.

The mobile care mediation agent detects the depressed individual's current mood state and presents suggestions to the depressed individuals or caregivers. The decision agent decides whether to display the corresponding suggestion to the depressed individual or caregiver after the depressed individual has completed the questionnaire. Screenshots of a notification sent to a caregiver are shown in Fig. 7. A suggestion is accompanied by a light bulb notification icon on the navigation bar at the top of the screen as depicted in Fig. 7 (left). An information bubble in HTML text anchored at the bottom of the screen as illustrated in Fig. 7 (middle) appears with a beep sound. The notification icon remains on the screen until the caregiver clicks to view the suggestion. Figure 7 (right) shows detailed descriptions of the depressed individual's current state and recommends a few support choices that the caregiver can perform in order to care for the depressed individual.

To update the image on the display screen, a user can use the phone camera to take a self-portrait, or select an image file that best represents his or her current situation. In Fig. 8, the caregiver shows an image of his or her breakfast with smiling emoticon, indicating that he or she just had a satisfying breakfast, while the depressed individual shows a self-portrait together with an emoticon. The updated image is also sent to the other party using the multimedia messaging service (MMS). A custom program was developed to send and receive the images. The photo and timestamp of the other party on the display are updated after the image is received.

The MMS Stack SDK was provided by WinWap technologies [29]. To send an MMS message, the device connects to GPRS and uploads the captured image to the MMS server of the telecom company. Since MMS uses the Wireless Application Protocol (WAP), the MMS server pushes the MMS notification message to the device. The custom MMS receiver has to register with the push router registration table to receive WAP push messages. The custom MMS receiver is invoked and connected to the GPRS after receiving notification for a new MMS message. The image can be downloaded from the MMS server as soon as the GPRS connection is established.
Fig. 7 Suggestion to caregiver: (left) light bulb notification icon, (middle) suggestion bubble anchored at the bottom of the screen, (right) suggestion content and choices
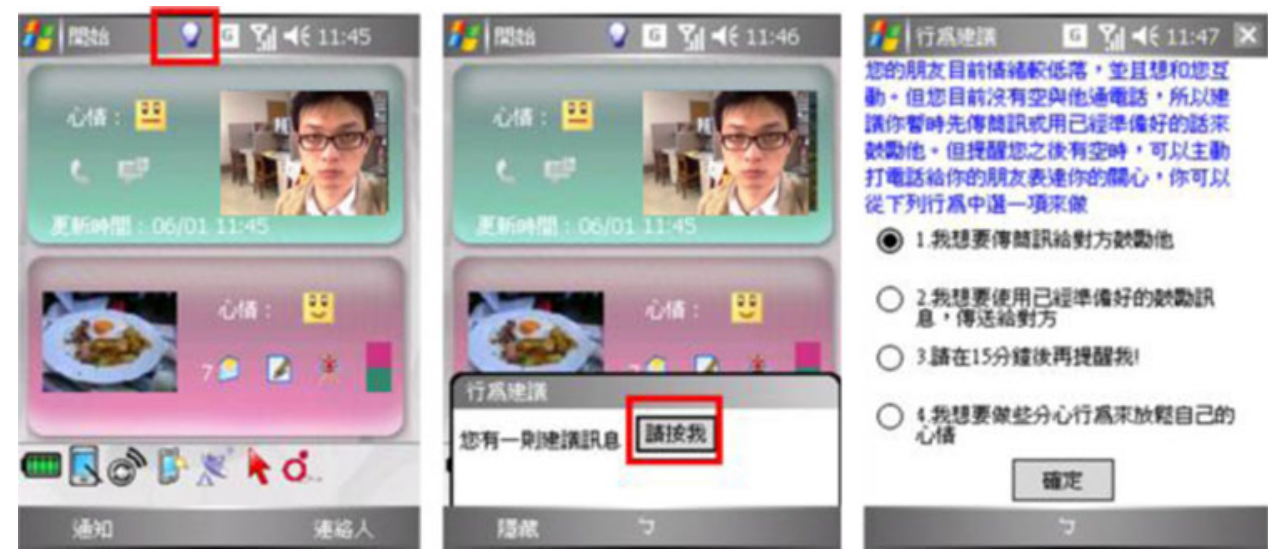


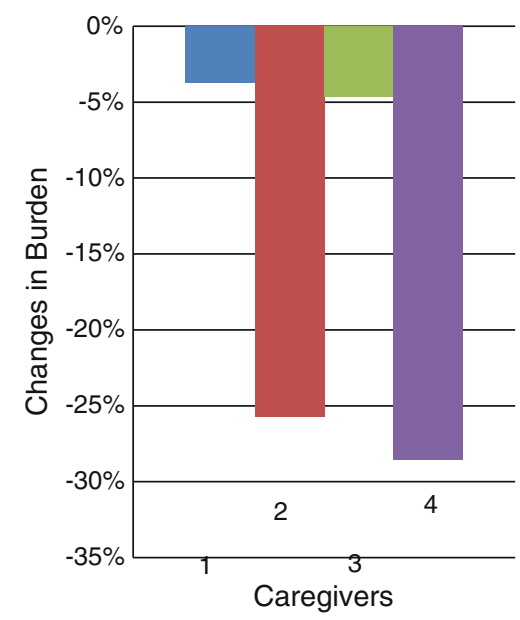

Fig. 8 Relative changes in caregiver burden

\section{Evaluation of the mobile care mediation system}

This user study was conducted in collaboration with the Department of Psychology of National Taiwan University (NTU) and involved four phases: (1) pre-ESM assessment for screening participants; (2) baseline ESM data collection for 1 week without adopting the care mediation tool between the depressed individuals and caregivers; (3) 2week experiment periods with the mobile care mediation tool, and (4) post-ESM assessment after using the mobile care mediation tool. The methodology of this user study is described as follows.

\subsection{Participants}

The participants were NTU students and were recruited via Internet advertisements. The study focused on students with dysphoric symptoms. Participants were screened using Beck Depression Inventory-II (BDI-II $>15)$, and by reassurance-seeking behavior (score $>$ mean $+0.5 \mathrm{SD}$ ). All of the participants that signed up took part in the preESM assessment, and those participants who were selected were requested to find a significant other as a caregiver to participate in this study. The caregiver was required to be a peer (such as an intimate friend or boyfriend or girlfriend) and not their senior (such as parents).

\subsection{Procedure}

The researchers met with the each of the selected participants and their caregivers to ask for their agreement to participate in 3-week study: 1 week for the baseline ESM assessment, and the following 2 weeks to use the mobile care mediation tool. Therefore, the participants were required to return twice during the study and were
Table 4 Total payment to participants for joining the study

\begin{tabular}{lll}
\hline & Dysphoric student & Caregiver \\
\hline Payment for participating & NT\$ 950 & NT\$ 700 \\
Reimbursement for SMS/MMS & NT\$ 550 & NT\$ 550 \\
Total & NT\$ 1,500 & NT\$ 1,250 \\
\hline
\end{tabular}

requested to place their own SIM cards into the experiment devices. Following the 1-week baseline period, the researchers held individual discussions with the participants about using their experience using the devices during the 1-week study. The participants were then told how the care mediation system worked. Participants were also informed that the mobile care mediation tool sends SMS and MMS messages to both parties to share information. As shown in Table 4, participants were reimbursed for the SMS/MMS messages sent using their phone numbers during the study. The post-ESM assessment was conducted when the participants returned the experiment devices.

\subsection{Measurements}

During the 1 week of ESM baseline study, the mood of the depressed individuals and caregivers was measured five times daily. Additionally, the mobile care mediation system was used to sample the overall mood status of the participants at the end of each day.

The following factors relating to the depressed individual were measured at the end of each day: (1) the extent to which they sought reassurance from the caregiver, (2) their feeling of being a burden to the caregiver, and (3) their level of understanding of the mood and availability of the caregiver during the day.

The following factors relating to the caregiver were measured at the end of each day: (1) the reassurance requested from the depressed individual, (2) their feeling of burden in caring for the depressed individual, and (3) their level of understanding of the mood and availability of the depressed individual during the day.

Identical questionnaires were used during the baseline and experiment periods of the mobile care mediation system. The experiment phase of the study included the interface, sensing, suggestion, and communication components of the system. Additionally, participants reported on their own moods and on the activities they performed during this phase. The questionnaires concerned the following:

- reductions in the caregiver's burden;

- reductions in reassurance-seeking behavior; and

- the degree of relation satisfaction between the depressed individual and the caregiver. 


\subsection{Results}

Five pairs of depressed individuals and caregivers participated in the study. All the participants completed the four phases of the study. One pair of participants was excluded from the evaluation results because the individual's depression level was much lower than the pre-ESM screening phase during the remainder of the 3-week study. The results in percentage changes before and after using the mobile care mediation tool are presented here. The changes could not be computed based on a global baseline because the participants were not all on the same baseline. Therefore, the percentage changes had to be based on the individual's pre-scores measured at the beginning of the study. The percentage was calculated as follows: (postscores-pre-scores)/pre-scores.

The evaluation results are described based on the four major measurements described in the user study design.

\subsubsection{Caregiver burden}

An involvement evaluation questionnaire (IEQ) [30] was utilized to measure the caregiver burden. Figure 8 shows the results. The burden of caregivers CG2 and CG4 was significantly decreased. Even though the burden of the other two caregivers was not significantly decreased, no caregivers experienced an increased burden at the end of the 3-week study.

\subsubsection{Reassurance-seeking behavior}

Reassurance-seeking behavior was measured in both depressed participants and caregivers. The depressive interpersonal relationships inventory-reassurance seeking subscale (DIRI-RS) [31] was utilized to measure reassurance-seeking (RS) behavior as felt by depressed participants, and reassurance-seeking behavior that caregivers perceived from depressed participants (Perceived RS). Figure 9 shows the results. Depressed individuals Dep1 and Dep2 exhibited a 40\% reduction in reassurance seeking, and the perceived reassurance seeking of their corresponding caregivers also decreased. However, depressed individual Dep3 and his corresponding caregiver experienced opposite outcomes. Caregiver CG4 exhibited a 33\% increase in perceived RS even though depressed individual Dep4 exhibited only slightly increased RS behavior.

\subsubsection{Relation satisfaction}

The relation satisfaction between depressed individuals and caregivers was measured by the relation assessment scale (RAS) [32]. Figure 10 shows the results. Each party estimated his or her relation satisfaction with the other party.

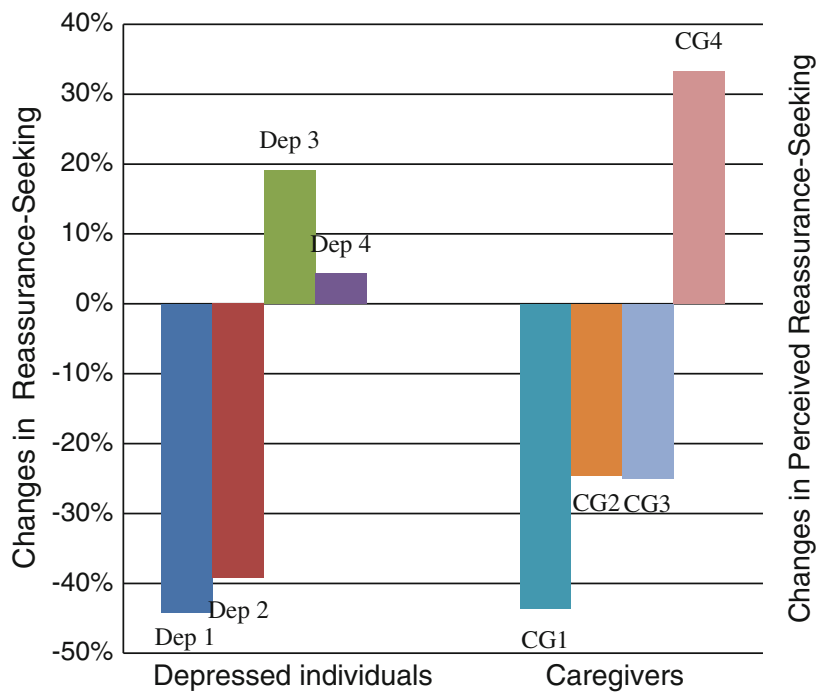

Fig. 9 Relative changes in the reassurance-seeking behavior experienced by depressed individuals (left) and caregivers (right) respectively

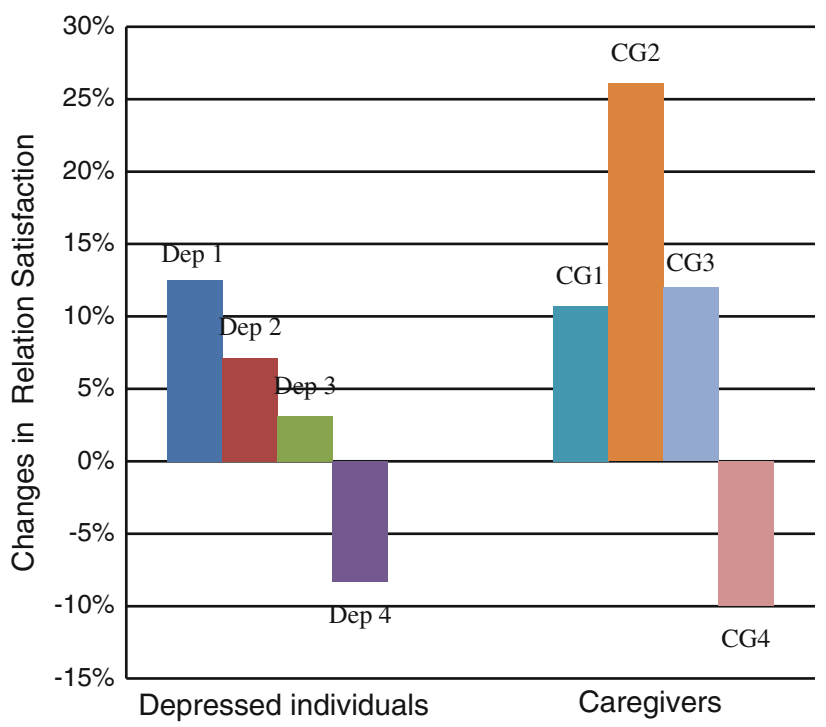

Fig. 10 Relative changes in the relation satisfaction experienced by depressed participants (left) and caregivers (right) respectively

The relation satisfaction of depressed individuals Dep1, Dep2, and Dep3 was enhanced, as was that of their corresponding caregivers CG1, CG2, and CG3. However, depressed individuals Dep4 and their corresponding caregivers had opposite outcomes.

\subsubsection{Summary}

Overall results were very positive for pairs 1 and 2. Not only did the depressed individuals reduce their reassuranceseeking behavior, but the caregiver burden was also 
decreased and their relationship satisfaction also increased. Their responses were encouraging, based on their use of our current prototype mobile care mediation tool.

While the reassurance-seeking behavior of pair 3 increased by $20 \%$, the corresponding caregiver's perceived reassurance-seeking declined by $25 \%$, and the caregiver burden did not change significantly $(-4.7 \%)$. The relation satisfaction felt by the caregiver increased by $12 \%$. The mobile care mediation tool cannot account for environmental factors that exacerbate depression. If the ruminative responses and reassurance seeking of the depressed participant increase due to external factors, then the mobile care mediation tool should ideally maintain, and not impair the interaction between the depressed participant and caregiver. Although the results of pair 3 looks promising, a long-term study is still required to discover whether the tool can help prevent the interaction quality from degrading when depression symptoms of the participants increase.

Although the overall results of pair 4 were not as encouraging as those of pairs 1 and 2, e.g., the reassurance seeking as perceived by the caregiver increased by $33 \%$ and the caregiver's relation satisfaction decreased by $10 \%$, the caregiver burden was reduced by $28.6 \%$.

Although this user study included only four pairs of participants and thus does not constitute statistically significant results, these results do indicate that the mobile care mediation system has the potential to enhance interaction between depressed individuals and caregivers. Additionally, psychologists can explore the interpersonal and emotional factors of the depressed individuals and caregivers with the assistance of technology.

\section{Discussion}

In this section, we review the current prototype design of the mobile care mediation system and consider future research directions. Three main observations are discussed below.

\subsection{The trade-off between the desire for more information and the burden of information input}

The initial goal of the information display is to enable participants to provide each other with up-to-date mood and availability information. The amount of information entered and presented in the display is designed to easily and quickly understandable, requiring few clicks, as to minimize the amount of interruption to caregivers or depressed individuals who may be busy with other activities. However, some participants commented that the amount of information presented in the display is insufficient to make an accurate assessment. For examples, when the caregiver sets his or her availability status to busy, the depressed individual may want to know whether this means that he or she should not contact the caregiver, or if he or she can still call the caregiver but only for perceived emergencies. At the same time, two caregivers also mentioned a lack of detailed information about the depressed individuals: "I only saw the sad face ... (which does) not provide sufficient clues for me to provide care ..." Thus there is a trade-off between the desire for more information and the burden of entering that information. That is, each party wants to know more about the other party, but he or she may not want the obligation to continuously update that information.

\subsection{Additional means of conveying emotion}

The system provides three ways for users to express their emotion: by filling out questionnaires, by setting an emoticon, and by capturing or showing a picture. Some participants commented that they disliked questionnaires because questionnaires limit the scope for conveying emotion and filling out the same questionnaires was boring. Therefore, depressed individuals suggested additional means that can better express their feeling or enable a caregiver to understand their emotion. For examples, animated emoticons could be used in addition to static emoticons. Using the camera on the phone to shoot a short video clip could be more expressive than a still photograph. Finally, a depressed individual could select and share music that best describes his or her emotion.

\subsection{Personalized suggestions}

The mobile care mediation system plays the role of an agent that suggests common distraction activities to depressed individuals and caring action to caregivers. However, since these suggestions are not personalized to individual preferences and context, some users rarely responded to them. To make these suggestions more relevant and appealing to users, the agent ought to include the input from psychologists and tailor suggestions to individual needs and preferences.

\section{Conclusion and future work}

We first presented a prototype of the ESM system and its evaluation. Second, we presented a prototype mobile care mediation system for depressed individuals and caregivers, where mobile phones are used to collect and share daily fluctuation of information, i.e., mood and availability, between depressed individuals and their caregivers. Additionally, the mobile phone also acts as an agent that 
suggests distraction exercises and caring action to both parties. A display interface was designed to present a quick summary of mood and availability information. User studies were undertaken to evaluate the phone-based ESM and the mobile care mediation systems. Results from a limited number of users suggested a higher compliance rate than that from the traditional paper-based ESM approach, reductions in caregiver burdens, and reduction in reassurance-seeking behavior from the depressed individuals.

Mobile technology can improve understanding and facilitate communication between depressed individuals and caregivers. In the future, we plan to improve the design of the mobile care mediation system to provide additional means of expressing mood and emotion and personalize recommendations for distraction or caring activities. A user study will also be undertaken for clinical depressed patients and their caregivers to evaluate the effectiveness of the mobile care mediation system.

Acknowledgments The study reported in this paper was supported in part by Research Grant from the National Science Council of Taiwan (NSC 96-2413-H-002-012-MY3).

\section{References}

1. World Health Organization (WHO) (2004) The World Health Report 2004: changing history, annex table 3: burden of disease in DALYs by cause, sex, and mortality stratum in WHO regions, estimates for 2002. WHO, Geneva

2. World Health Organization (WHO) (2002) Global burden of disease (GBD) 2002 estimates. Estimates of numbers of prevalent cases for selected causes, by sex and WHO region, estimates for 2002. WHO

3. The Hindustan Times (2001) One out of four mentally ill

4. Department of Health Executive Yuan (2005) Statistics of causes of death. ROC (Taiwan)

5. Oquendo MA et al (2001) Ethnic and sex differences in suicide rates relative to major depression in the United States. Am J Psychiatry 158:1652-1658

6. Yang HJ (2002) A follow-up study of depressive disorders and depressive symptoms in adolescents. National Taiwan University, Taipei, Republic of China

7. Proudfoot J (2004) Computer-based treatment for anxiety and depression: is it feasible? Is it effective? Neurosci Biobehav Rev 28:353-363

8. Purves B, Purves D (2007) Computer based psychotherapy for treatment of depression and anxiety. In: 14th annual IEEE international conference and workshops on the engineering of computer-based systems. Tucson, Arizona, USA. IEEE Computer Society, Washington, DC

9. Warmerdam L, van Straten A, Cuijpers P (2007) Internet-based treatment for adults with depressive symptoms: the protocol of a randomized controlled trial. BMC Psychiatry 7:72

10. Herman S, Koran L (1998) In vivo measurement of obsessivecompulsive disorder symptoms using palmtop computers. Comput Human Behav 14:449-462
11. Przeworski A, Newman MG (2004) Palmtop computer-assisted group therapy for social phobia. J Clin Psychol 60:179-188

12. Joiner T, Coyne JC, Blalock J (1999) On the interpersonal nature of depression: overview and synthesis. In: Joiner T, Coyne JC (eds) The interactional nature of depression. pp 3-19

13. Joiner $\mathrm{T}$ et al (2002) Perceived burdensomeness and suicidality: two studies on the suicide notes of those attempting and those completing suicide. J Soc Clin Psychol 21(5):531-545

14. Pettit JW, Joiner T (2006) Depression chronicity: perspectives on forms and reasons, in chronic depression: interpersonal sources. In: Pettit JW, Joiner T (eds) Therapeutic solution. American Psychological Association, Washington, DC, pp 3-11

15. Day M, Rosenberg J, Sugano H (2000) A model for presence and instant messaging. RFC 2778

16. Debbabi M, Rahman M (2004) The war of presence and instant messaging: right protocols and APIs. In: IEEE consumer communications and networking conference. Las Vegas, Nevada, USA

17. Intille SS et al. (2003) A context-aware experience sampling tool. In: ACM CHI 2003 extended abstract. ACM Press, New York

18. Froehlich $\mathbf{J}$ et al. (2007) MyExperience: a system for in situ tracing and capturing of user feedback on mobile phones. In: ACM Mobisys. San Juan, Puerto Rico

19. Mulder I, ter Hofte GH, Kort J (2005) SocioXensor: measuring user behavior and user experience in context with mobile devices. In: Measuring behavior 2005. Wageningen, The Netherlands

20. Carter S, Mankoff J, Her J (2007) Momento: support for situated Ubicomp experimentation. In ACM CHI 2007. San Jose, California, USA

21. Mamykina L et al. (2008) MAHI: investigation of social scaffolding for reflective thinking in diabetes management. In ACM CHI 2008. Florence, Italy

22. Mynatt ED et al. (2001) Digital family portraits: supporting peace of mind for extended family members. In ACM CHI. Seattle, Washington, USA

23. Consolvo S, Soessler P, Shelton B (2004) The carenet display: lessons learned from and in home evaluation of and ambient display. In: Ubicomp. Nottingham, UK

24. Consolvo $\mathrm{S}$ et al. (2008) Activity sensing in the wild: a field of trial of UbiFit garden. In ACM CHI 2008. Florence, Italy

25. Chang YC et al. (2008) Playful toothbrush: UbiComp technology for teaching tooth brushing to kindergarten children. In ACM CHI 2008. Florence, Italy

26. de Sa M, Carrico L, Antunes P (2007) Ubiquitous psychotherapy. IEEE Pervasive Comput 6:20-27

27. Beck AT, Steer RA, Brown GK (1996) Manual for the beck depression inventory-II. Psychological Corporation, IEEE Pervasive Computing

28. Watson D, Clark LA, Tellegen A (1988) Development and validation of brief measures of positive and negative affect: the PANAS scale. J Personal Soc Psychol 54:1063-1070

29. WinWap Technologies. Available from: http://www.winwap.com/

30. van Wijngaarden B et al. (2000) Caregiving in schizophrenia: development, internal consistency and reliability of the Involvement Evaluation Questionnaire-European Version. EPSILON Study 4. European Psychiatric Services: inputs linked to outcome domains and needs. Br J Psychiatry 177:s21-s27

31. Joiner TE, Metalsky GI (1995) A prospective test of an integrative interpersonal theory of depression: a naturalistic study of college roommates. J Personal Soc Psychol 69:778-788

32. Hendrick SS, Dicke A, Hendrick C (1998) The relationship assessment scale. J Soc Pers Relat 15:137-142 